\title{
Computational studies of mycorrhizal protein: GiHsp60 and its interaction with soil organic matter
}

\author{
Dipti Mothay and K. V. Ramesh* \\ Department of Biotechnology, Jain (deemed to be University), School of Sciences, Jayanagar 3rd Block, Bengaluru 560 011, India
}

\begin{abstract}
This study uses homology modelling and molecular docking approaches to explore the binding mechanism of glomalin-related soil protein from Rhizophagus irregularis (GiHsp60) with soil organic matter (SOM) and the role played by soil protein in the sequestration of common soil pollutants. Conserved domain analysis reveals that GiHsp60 belongs to chaperonin-like super-family having binding sites for ATP/ $\mathrm{Mg}^{2+}$. Threedimensional model of GiHsp60 was reasonably good based on reports generated by different validation servers. Docking results suggest that Van der Waals force is primarily responsible for the interaction between GiHsp60 and SOM. The study also reveals the role played by GiHsp60 in the sequestration of different soil pollutants.
\end{abstract}

Keywords: Docking studies, homology modelling, heat shock protein, mycorrhizal fungi, soil pollutants.

SoIL organic matter (SOM) is a complex, naturally occurring material that plays a critical role in soil fertility, global carbon cycle and the fate of pollutants in the soil. SOM influences structure and productivity as well as aggregate stability of the soil ${ }^{1}$, which is mediated through a number of biotic and abiotic factors ${ }^{2}$. The mycorrhizal fungi being an important biotic factor among soil microbial communities, play a critical role in soil aggregate formation and stabilization ${ }^{3}$. Wright and Upadhyaya ${ }^{4}$ while studying mycorrhizal fungi, discovered a soil protein known as glomalin ('soil glue'). Due to difficulty in isolation and the presence of potentially contaminating compounds in soil-extracted materials, characterizing glomalin has proved to be difficult ${ }^{5}$. While the term 'glomalin' is reserved for a purified gene product ${ }^{4}$, it is operationally defined as glomalin-related soil protein (GRSP).

Gadkar and Rillig ${ }^{6}$ using various experimental techniques provided the first evidence for identifying GRSP protein in Rhizophagus irregularis (abbreviated as GiHsp60). Their studies helped conclude that GiHsp60 is a putative homolog of heat shock protein 60 (Hsp60). This protein when released into the soil during hyphal

*For correspondence. (e-mail: kv.ramesh@jainuniversity.ac.in) turnover becomes a component of SOM and contributes to physical stability of the soil ${ }^{7}$. GiHsp60 has been gaining increasing attention not only for its role in maintaining soil fertility, but also for sequestering heavy metals ${ }^{8}$.

While many correlations between GiHsp60 and agronomic indicators have been reported, structure and molecular characterization of GiHsp60 remains unclear ${ }^{9}$. Thus, efforts to manipulate this important soil protein have remained compromised till date. Any attempt made towards understanding the structural details of this soil protein will boost agricultural productivity. Our recently conducted evolutionary study on GiHsp60, explains the possibility of increased genetic differentiation experienced by $h s p 60$ (gene) of $R$. irregularis ${ }^{10}$. As an extension of this work, the twin objectives of the present study are: (i) modelling the 3D structure of GiHsp60 and (ii) molecular docking studies between SOM with GiHsp60 and soil pollutants, namely benzochrysene - a polyaromatic hydrocarbon and chromium (III).

\section{Materials and methods}

\section{Identification of conserved motifs}

The NCBI CDD tool was used to predict duplication of conserved regions (domains) for GiHsp60 (ref. 11). Protein sequence for GiHsp60 along with one of its potential PDB templates was submitted to ProDom server for detecting the presence of any domains ${ }^{12}$. To identify novel sequence motifs of GiHsp60, 52 Hsp60 sequences (identified based on the multiple sequence alignment profile) ${ }^{13}$ along with sequence information of GiHsp60 were submitted to MEME program version 4.12.0 (ref. 14). Amino acid sequence of GiHsp60 and sequences of ten most closely related Hsp60 taxa were further submitted to the PRATT server ${ }^{15}$

\section{Prediction of secondary structural features and $\mathrm{N}$-glycosylation profile for GiHsp60}

Secondary structural features (helices $(H)$, sheets $(E)$, coils (C)) of GiHsp60 sequence, including solvent 
accessibility and H-bonding were predicted using JOY server $^{16}$. The NetNGlyc ${ }^{17}$ server was used to find the $\mathrm{N}$ glycosylated sites of GiHsp60. The $N$-glycosylation potential $>0.5$ was considered as cut-off value for sequences.

\section{Three-dimensional structure prediction and validation}

For building the 3D model of GiHsp60, the target sequence information was submitted to SWISS-MODEL ${ }^{18}$. The 3D model was then subjected to loop refinement based on reports generated by ERRAT ${ }^{19}$. Loop regions of the model showing a high percentage of error were refined through ModLoop server ${ }^{20}$. Energy minimization of the model was carried out using DeepView package ${ }^{21}$. The model was subsequently validated using Verify $3 \mathrm{D}^{22}$, ProSA $^{23}$ and PROCHECK ${ }^{24}$ servers. The final structure was visualized and analysed using the SPDBV 4.10 $\operatorname{program}^{21}$.

\section{Docking studies of SOM with GiHsp60}

The SOM model proposed by Schulten and Schnitzer ${ }^{25}$ was downloaded (mol format) from 'The Virtual Museum of Minerals and Molecules ${ }^{\text {TM }}{ }^{26}$. Although the model was geometrically optimized based on molecular mechanics calculations using HyperChem software, in the present study it was reoptimized using AM1 (Austin model), a semi-empirical method as model chemistry using Gaussian 09 program $^{27}$. After successful completion of geometry optimization, total energy and convergence details were recorded from the log file of reoptimized SOM (rSOM) model. These log files were later visualized using GaussView ${ }^{28}$. Here, we have used both the original SOM (native) and the rSOM models for docking studies.

\section{Generating parameter and coordinate files for the $3 \mathrm{D}$ model of GiHsp60}

Before the docking exercise, for checking any anomalies in the 3D model of GiHsp60, the structure was processed using tLEaP program implemented in AMBER10 (ref. 29). Initially, the GiHsp60 model was reduced by adding $\mathrm{H}$ using the Chimera package ${ }^{30}$. Using the Leap program, the respective topology (prmtop) and coordinate files (rst, inpcrd) for the GiHsp60 model were generated and later converted to pdb files with the ambpdb command of AMBER, which was used as input file for docking.

\section{Molecular docking}

SOM and GiHsp60: Docking calculations were carried out with Dock6 program installed in SGI AltixUV10 (ref. 31). Two independent rigid docking exercises were car- ried out: GiHsp60 (receptor) with native SOM (ligand) followed by docking with r-SOM (ligand). All default sets of dock parameters were retained for both docking exercises. Final grid_score and grid_vdw were recorded for both docking exercises. The docked ligands were visualized along with the receptor using either Chimera or PyMOL package ${ }^{32}$. The PDB2PQR server was used to generate pqr and $\mathrm{dx}$ files for GiHsp60 (ref. 33). Using this information, PyMOL plugin 'APBS' program was run to display electrostatic potential surface and solvent accessible surface area for the GiHsp60 model.

SOM and soil pollutants: To explain adsorption/ desorption of soil pollutants onto the surface of SOM, docking studies were concluded between the r-SOM model (receptor) and few selected common soil pollutants such as benzochrysene - a polyaromatic hydrocarbon and chromium ion $\left(\mathrm{Cr}^{3+}\right)$ using AUTODOCK tools. Structural coordinates of the free form of benzochrysene (http:// www.rcsb.org/ligand/BC) and unbounded chromium ion (http://www.rcsb.org/ligand/CR) were downloaded from PDB and subsequently saved in pdb format using GaussView. Gridbox centre was set to coordinates -6.539 , -1.297 and 143.202 in $x, y$ and $z$ respectively. Polar $H$ charges of the Gasteiger-type were assigned and nonpolar $\mathrm{H}$ atoms were merged with the carbons, and internal degrees of freedom and torsions were set. Default settings were used for all other parameters. PyMOL was used to visualize the binding interactions of $\mathrm{r}-\mathrm{SOM}$ with soil pollutants.

\section{Results}

\section{Analysis of conserved domains and sequence motifs}

The CDD server maps residues that constitute ATP/ $\mathrm{Mg}^{2+}$ binding sites, hinge region, ring oligomerization interface and stacking interaction for the GiHsp60 sequence. Results from the ProDom server suggest that this sequence has 17 domains sharing several common domains with that of 5DA8 (PDB template).

Among the ten putative conserved motifs in the GiHsp60 sequence identified by the MEME server, eight motifs (1-3, 5-8 and 10) appeared to be highly conserved while the remaining two motifs (4 and 9) were partially conserved. Additional analysis using ScanProsite identified a smaller motif of 12 residues ("AAVEEGTVPGGG') having the function of chaperonin60 (cpn60). This conclusion was arrived at based on signature consensus pattern (shown below) of cpn60 generated by the PRATT server for GiHsp60 and ten other homologous taxa

$$
\mathrm{A}-[\mathrm{AS}]-\{\mathrm{L}\}-[\mathrm{DEQ}]-\mathrm{E}-\{\mathrm{A}\}-\{\mathrm{Q}\}-\{\mathrm{R}\}-\mathrm{X}-\mathrm{G}(2)-[\mathrm{GA}] .
$$

The combined results of CDD, MEME and ScanProsite for GiHsp60 showed that among four different types of 
functional sites, both motifs 8 and 9 had three distinct functionally important residues. ScanProsite also identified additional motif regions for GiHsp60.

\section{Secondary structure prediction for GiHsp60 sequence}

Based on the output from the Joy server (Supplementary Figure 1), nearly $76 \%$ residues for GiHsp60 appear to be in solvent-accessible region followed by $24 \%$ residues in the solvent-inaccessible region. Further, secondary structural analysis showed more number of residues forming ' $\alpha$-helix' $(\sim 4 \%)$ than $\beta$-strand $(\sim 26 \%)$, with $2 \%$ of residues forming $3_{10}$ helix. While nearly $15 \%$ of the residues accounted for $\mathrm{H}$-bond to main chain carbonyl, $4 \%$ contributed to H-bond to main-chain amide.

\section{N-linked glycosylation profile for GiHsp60}

The $N$-linked glycosylation sites predicted by NetNGlyc were located at residues 115, 438 and 465 for GiHsp60, which crossed the set threshold value of 0.5 (Supplementary Figure 2).

\section{Three-dimensional model of GiHsp60}

The SWISS-MODEL was successful in generating a 3D structure for GiHsp60 using crystal structure of chaperonin GroEL from 5DA8 as a template. Quality factor of the initial model evaluated by ERRAT increased from $90.69 \%$ to $92.23 \%$ (Supplementary Figure 3), and energy of the model decreased from $-22,0638.01$ to $-21,330.072 \mathrm{~kJ} \mathrm{~mol}^{-1}$ upon minimization. Verify3D (Supplementary Figure 4 ) showed that $85.74 \%$ of residues of the GiHsp60 model had an average 3D-1D score $\geq 0.2$ which represents a good score, suggesting high compatibility of the atomic model with its amino acid sequence.

Validation of the loop-refined GiHsp60 model using Ramachandran plot revealed that $93 \%$ of the residues were in the most favoured regions, followed by $6.1 \%$ in the additional allowed regions, $0.4 \%$ in the generously allowed regions and $0.4 \%$ in the disallowed regions. Overall G-factor for the predicted structure was -0.13 (Supplementary Table 1). The G-factor provides a measure of the normalcy of stereo-chemical property of a protein model. Values below -0.5 show unusual stereochemical property, while those below -1.0 show highly unusual property. Since the G-value obtained for the predicted model in the present study was not less than -0.5 , it indicates satisfactory quality. Main-chain parameter plot statistics suggested that the overall quality of the predicted model is better. ProSA energy plot revealed negative energy distribution pattern being scored by the residues for the predicted structure (Supplementary Fig- ure 5) with a $z$ score of -11.17 , which is within the range of scores reported for NMR-derived structure for the native protein of similar size. The Chimera package was able to superimpose the refined 3D model of GiHsp60 onto the crystallographic structure of Hsp60 from $C$. tepidum (PDB id: 5DA8_A). Root mean square deviation of $\mathrm{C}_{\alpha}$ atoms between the target (GiHsp60) and template (5DA8_A) computed was $0.35 \AA$.

\section{Docking and molecular interaction studies of SOM with GiHsp60 and soil pollutants: benzochrysene and chromium}

Docking of native SOM with GiHsp60 resulted in dock energy (grid_vdw score) of $-3.54 \mathrm{kcal} \mathrm{mol}^{-1}$. Upon reoptimization, the studied 3D model of r-SOM recorded an energy value of $-15.7 \times 10^{3} \mathrm{~kJ} \mathrm{~mol}^{-1}$ (Figure 1). Although molecular docking was performed with all the clusters using box length as $8 \AA$, rigid docking between GiHsp60 and r-SOM was successful when the box margin was increased to $16 \AA$ (Figure 2). Using this as the box dimension; among all the eight clusters generated, grid_vdw score value of $-8.57 \mathrm{kcal} \mathrm{mol}^{-1}$ of cluster 1 was the best; it was better compared to the docking energy of native SOM with GiHsp60. Analysis of the docked complex suggests that r-SOM was docked into the cavity of the GiHsp60 protein. Upon closer examination, the putative binding site of GiHsp60 representing ' $\operatorname{asn}^{67}, \operatorname{gln}^{41},{ }^{210}$, $\mathrm{glu}^{66}, \mathrm{leu}^{266}, \mathrm{tyr}^{43,202,205}$, appeared to be in close proximity to SOM model. Based on the APBS-generated solvent accessible surface, GiHsp60 is found to be rich in residues contributing towards negative potential. Docking results further suggest that various groups of the SOM model (carboxylic, phenolic, alcoholic, quinonic, ketone, methoxyl, saccharides, proteinaceous material and hydrating water molecules) appear to interact with GiHsp60, thus contributing to the structural stability of SOM (Figure $3 a$ and $b$ ). From Figure $4 a$ and $b$, hydrophilic interactions appear to be predominant than hydrophobic interactions between different atoms of SOM and active-site residues of GiHsp60; both these interactions favoured $\mathrm{H}$-bonding of varying lengths between the ligand (SOM) and receptor (GiHsp60), resulting in the structural stability of the GiHsp60-SOM complex.

Analysing the molecular interactions of benzochrysene and chromium on the surface of SOM (Figure $5 a$ and $b$ ) and correlating this with our earlier reported docking output of SOM with GiHsp60, it appears that this protein interferes at the binding site of SOM with soil pollutants, thereby being responsible for displacing the pollutants from binding onto the surface of SOM. This is further substantiated by visual inspection of the binding region of the SOM structure, wherein the GiHsp60 protein occupies an extended surface area of the SOM model, thereby masking the potential binding sites of soil pollutants. 


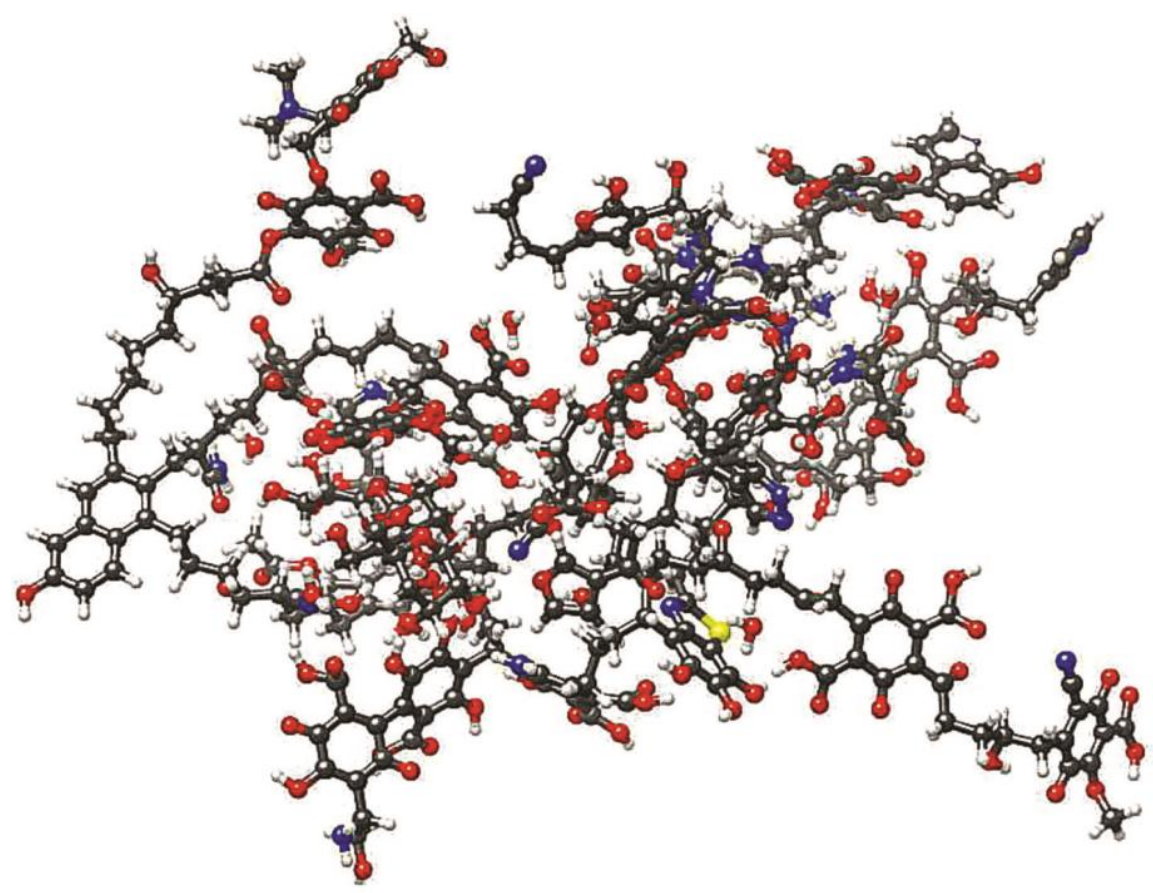

Figure 1. Reoptimized soil organic matter (r-SOM) model ${ }^{25}$ based on AM1 semi-empirical method using GAUSSIAN software. The log file generated after the successful completion displayed: SCF done; $* \mathrm{E}(\mathrm{RAM} 1)=-1.57 \times 10^{4} \mathrm{~kJ} \mathrm{~mol}^{-1}$ after 111 cycles; convergence $=0.2279 \mathrm{D}-08$. The element colours are: carbon (grey), hydrogen (white), oxygen (red), nitrogen (blue) and sulphur (yellow). (*E (RAM1) refers to energy for restricted Austin Model.)

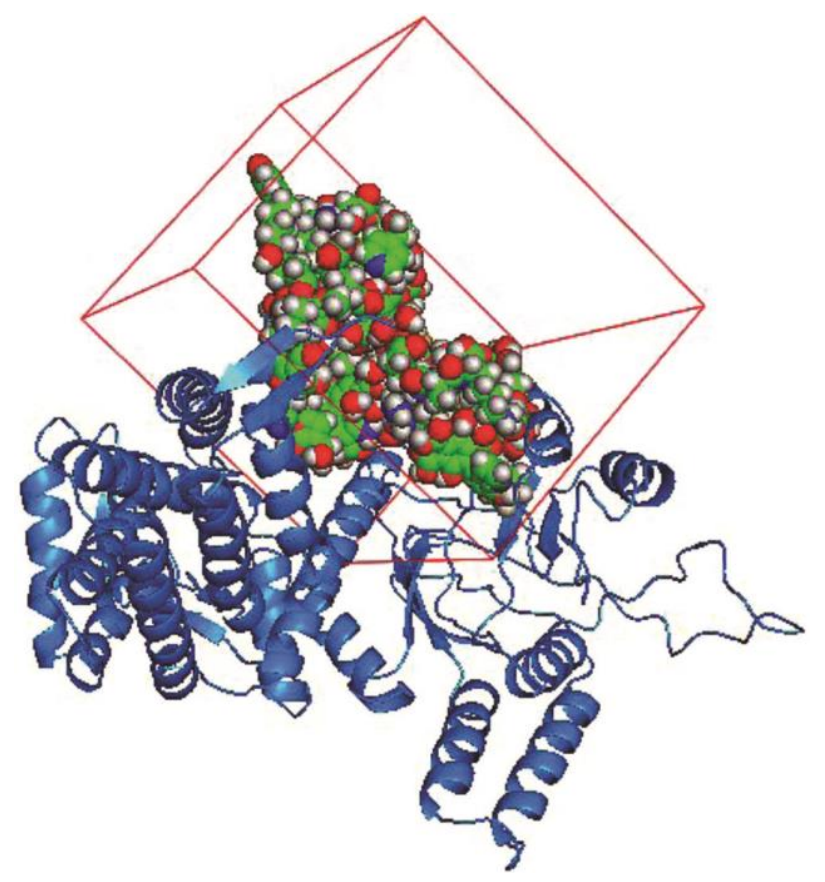

Figure 2. Docking of the best pose of r-SOM (ligand) onto the 3D model of GiHsp60 (receptor) using rigid docking method as implemented in DOCK software (grid_vdw $=-8.57 \mathrm{kcal} \mathrm{mol}^{-1}$ ). While the secondary structure of the receptor is represented as a cartoon (red $=$ helix, yellow $=$ sheet, green $=$ coil $)$, the ligand is shown as spheres. Box dimension of $x=38.487, y=47.249$ and $z=41.766$ (red colour) enclosing the docked complex was generated with length set to $16 \AA$ in the input file showbox.in. The image was generated using PyMOL package.
In the absence of any experimental evidence to date, this theoretical study elucidates the 3D structure of GiHsp60, followed by docking studies explaining Van der Waals interaction as one of the possible mechanisms by which the soil protein is adsorbed onto the surface of the SOM model. The study also highlights the role of this important mycorrhizal protein in sequestering soil pollutants.

\section{Discussion}

Despite much work performed on GiHsp60, there are significant gaps in our knowledge about this important soil protein. Thus, using homology modelling and docking approaches, the present study aims to predict the 3D structure of GiHsp60, understand its interaction with r-SOM and explain the role of GiHsp60 in the adsorption/desorption phenomenon of common soil pollutants.

CDD analysis revealed that GiHsp60 belonged to the chaperonin-like super-family having binding sites for $\mathrm{ATP} / \mathrm{Mg}^{2+}$, which is in line with the observations made by Purin and Rillig 5 . Secondary structural analysis revealed that the GiHsp60 sequence had more number of residues forming helices than sheets. Also, the bulk of residues being present in the solvent-accessible region may additionally contribute towards higher hydrophobic free energies due to the transfer of non-polar residues 


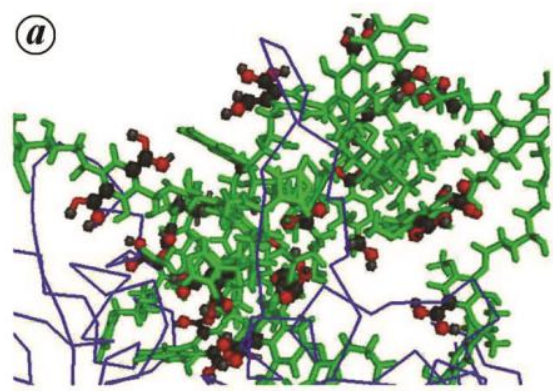

(i) Carboxylic groups

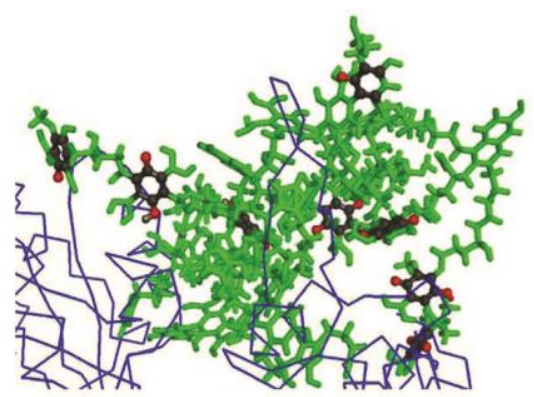

(iv) Quinomic groups

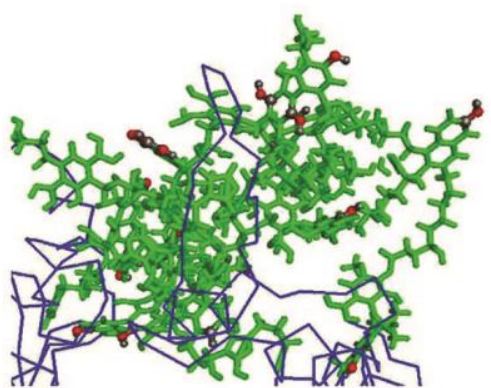

(ii) Phenolic groups

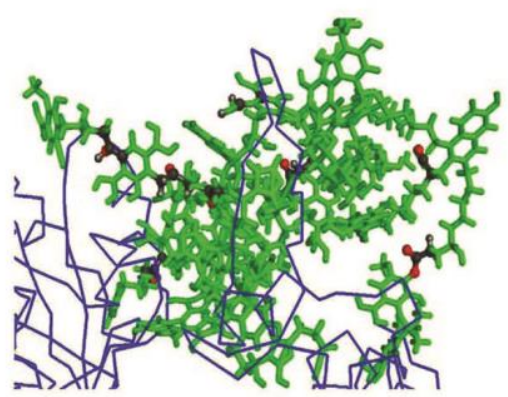

(v) Ketone groups

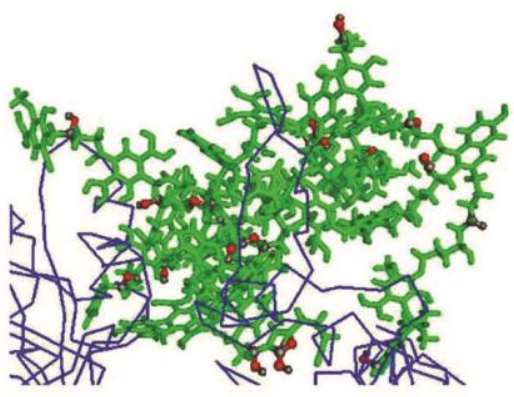

(iii) Alcoholic groups

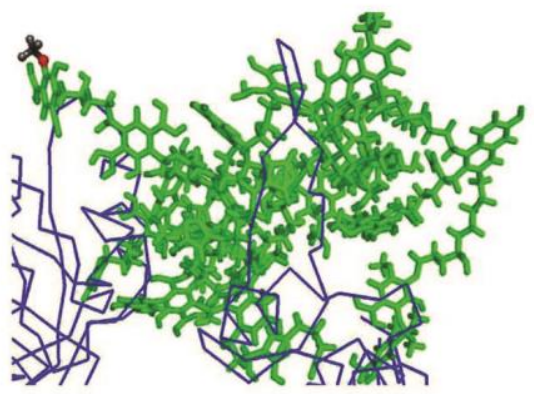

(vi) Methoxyl groups

(b)

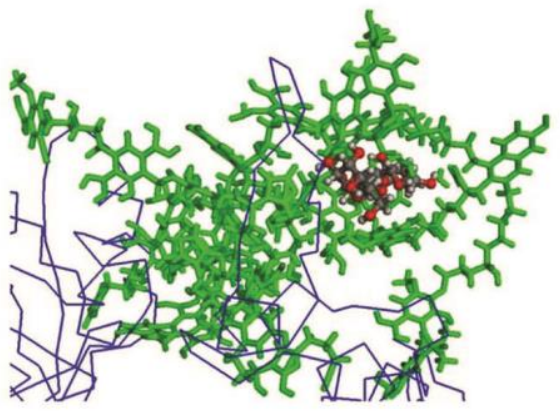

(vii) Saccharides (sugars)

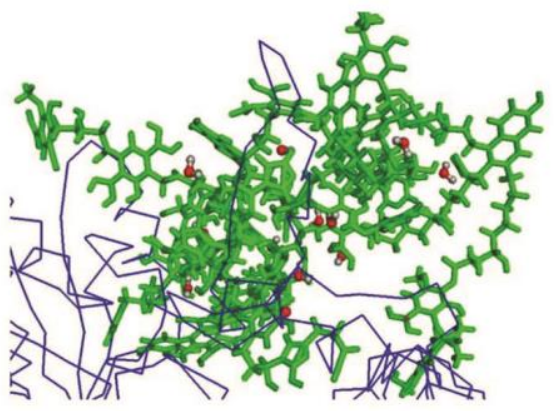

(ix) Water

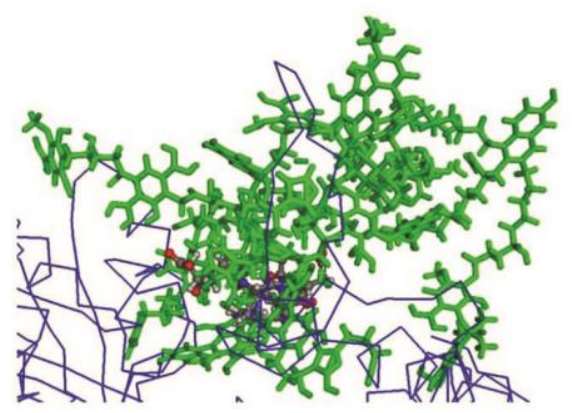

(viii) Proteinaceous material

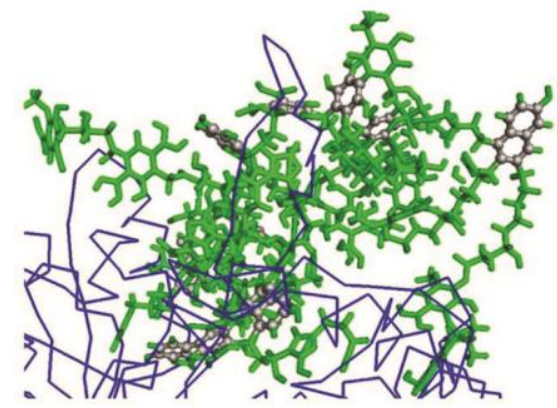

(x) Aromatic carbon

Figure 3a, b. Representation of various groups ((i)-(x)) of r-SOM (green colour) interacting with GiHsp60 (blue ribbon). The element colours of the various groups of SOM are: carbon (grey), hydrogen (white), oxygen (red) and nitrogen (blue). Image was generated using PyMOL package. (Note: Various groups of SOM interacting with GiHsp60 have been traced by comparing them with native SOM available in 'The Virtual Museum of Minerals and Molecules ${ }^{\mathrm{TM}}$; https://virtual-museum.soils.wisc.edu/.)

from an aqueous environment to the interior of the proteins ${ }^{34}$. In support of this observation, Lin et al. ${ }^{35}$ have demonstrated hydrophobic interactions as a driving force for the association of substrates with GroEL (the bacterial homolog of Hsp60). The $N$-linked glycosylation sites identified were also in accordance with those identified by Gadkar and Rillig6.

Quality of the 3D structure of GiHsp60 using 5DA8 as the template was reasonably good based on validation reports generated by various servers. In the absence of 
(a)

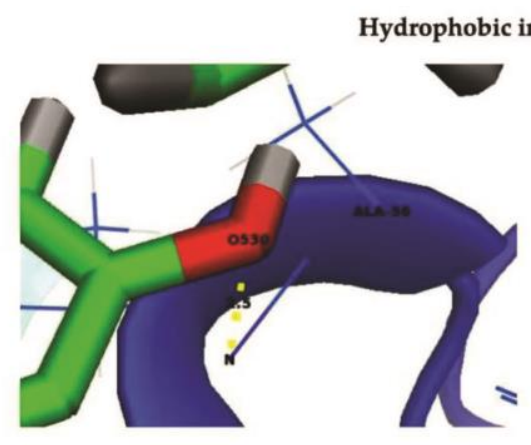

(i) SOM_O ${ }^{530}$ :: N_ala ${ }^{56}{ }_{-}$GiHsp60

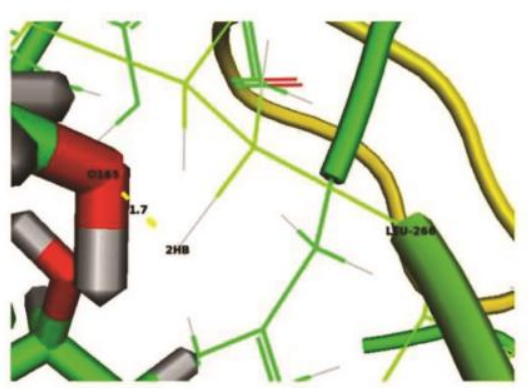

(ii) SOM $(-\mathrm{OH}) \_\mathrm{O}^{165}$ :: 2HB_leu ${ }^{266} \_$GiHsp60

Hydrophilic interactions
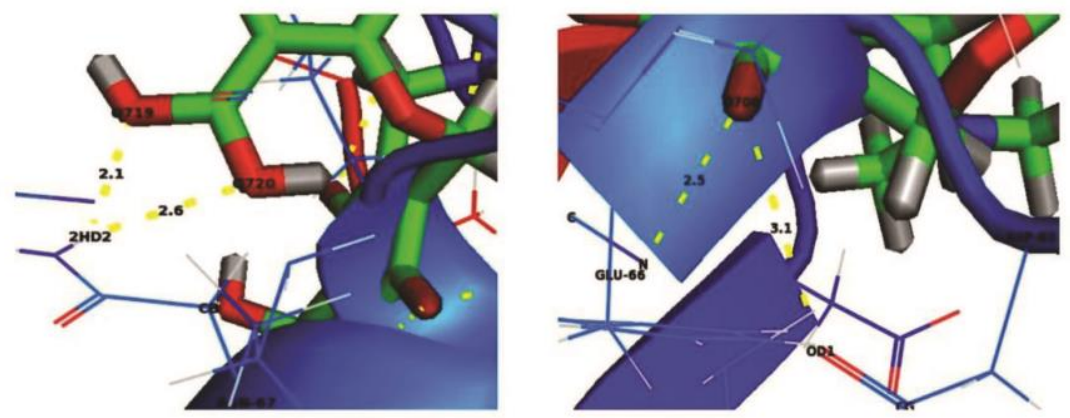

(b)

(iii) SOM (-COOH)_ $\mathrm{O}^{79} \& \mathrm{O}^{720}:$ 2 $2 \mathrm{HD} 2 \_$asn ${ }^{67} \_$GiHsp60

(iv) $\operatorname{sOM}(Q G) \_\mathbf{O}^{700}:$ : N \& OD1_glu ${ }^{66} \_$GiHsp60
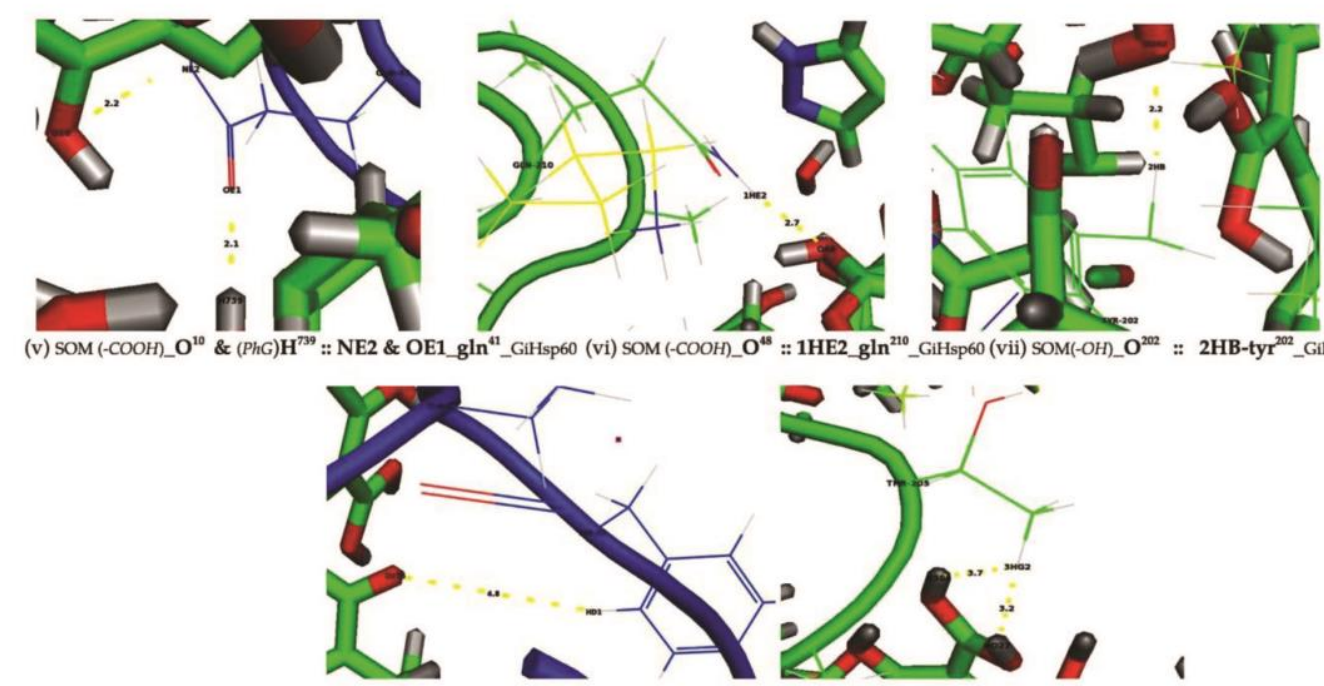

(viii) SOM (QG)_O $\mathbf{O}^{255}$ :: HD1_tyr ${ }^{3}{ }^{3}$ GiHsp60

(ix) SOM (-COOH)_ $\mathbf{H}^{506} \& \mathbf{H}^{0 / 2}$ :: 3HG2_tyr ${ }^{205} \_$GiHsp60

Figure $4 \boldsymbol{a}, \boldsymbol{b}$. Hydrophobic and hydrophilic interactions between different atoms (belonging to various groups) of SOM and active site residues of GiHsp60. While SOM model is represented as a stick, GiHsp60 model is shown as a cartoon. Abbreviations within parentheses: $-\mathrm{COOH}$, Carboxylic group; $-\mathrm{OH}$, Hydroxyl group; QG, Quinonic group and PhG, Phenolic group. The image was generated using PyMOL package.

any experimental structures, there is ample evidence cited in the literature to illustrate the usefulness of theoretical structures of HSPs to understand the structure-functional relationship ${ }^{36}$.

Protein adsorption at solid surfaces such as SOM plays a key role in many natural processes. Docking of r-SOM onto GiHsp60 revealed that these two molecules were docked with a grid_vdw score value of $-8.57 \mathrm{kcal} \mathrm{mol}^{-1}$. This suggests that collective Van der Waals force is primarily responsible for interaction between GiHsp60 and r-SOM. Individual Van der Waals bonds are shortlived, but this form of bonding can achieve greater permanence if many such linkages are formed across both the adsorbing substance and the surface because, at any 


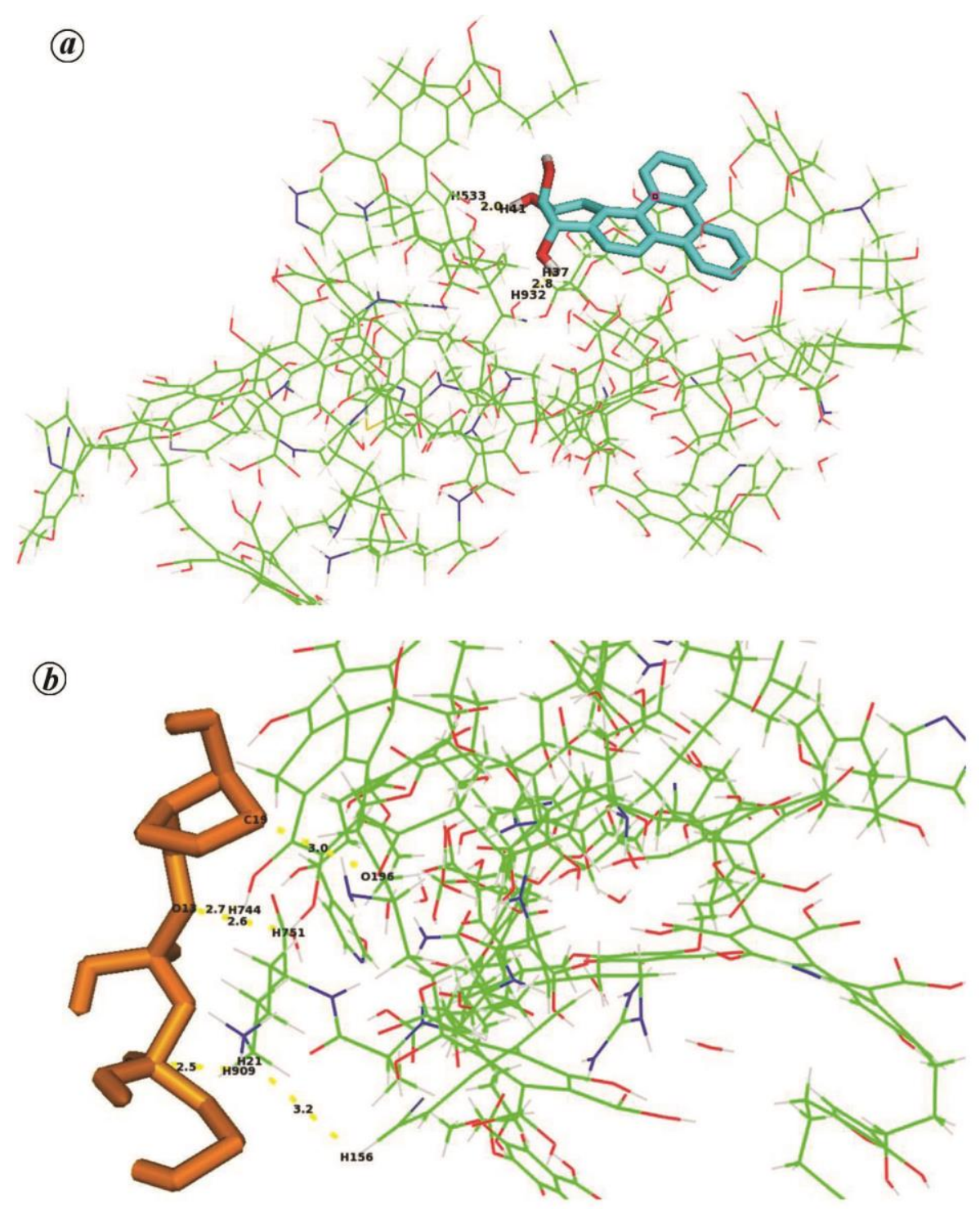

Figure 5. Docking and molecular interactions of $(\boldsymbol{a})$ benzochrysene and $(\boldsymbol{b})$ chromium ion $\left(\mathrm{Cr}^{3+}\right)$ in the SOM model. The structure was visualized using PyMOL package.

given time, sufficient Van der Waals bonds can suffice to prevent desorption. This is sometimes important for the binding of high molecular weight organic matter such as proteins to the surfaces ${ }^{37}$.

Molecular interaction studies revealed hydrophilic interactions to be predominant than hydrophobic interactions between different atoms of r-SOM and active site residues of GiHsp60. Further, these interactions were stabilized with $\mathrm{H}$-bonds of varying lengths between the ligand (r-SOM) and the receptor (GiHsp60), presumably resulting in the structural stability of the GiHsp60-rSOM complex. The present study suggests tyrosine of GiHsp60 as one of the critical active site residues interacting with SOM. Among the various groups of SOM interacting with selective residues of $\mathrm{GiHsp60,}-\mathrm{COOH}$ seems to be of greater significance followed by the alcoholic, phenolic and quinonic groups. This is in line with the observations made by Perry et al. ${ }^{38}$, who reported that in addition to alcohol/phenol groups, carboxylate group of organic matter primarily interacts with mineral interfaces.

Additional docking studies were carried out to examine the role of GiHsp60 in the sequestration of different soil pollutants. Output from these studies suggests that a larger surface area of the SOM structure is accessible for interaction with GiHsp60, thus being responsible for desorption of soil pollutants. In the recent past, several experimental studies have reported the contribution of GiHsp60 towards sequestration of potential toxic elements $^{39}$. Both complexation and adsorption mechanisms are involved in the linking of metals/organic pollutants by $\mathrm{SOM}^{40}$. Adsorption of polyaromatic hydrocarbon (PAHs) onto SOM, fulvic acid (FA) and humic acid (HA) 
depends on the properties of PAHs and the SOM models. Preliminary docking studies carried out by Saparpakorn et $a l .{ }^{41}$ have revealed important binding modes to FA, HA and SOM, and thereby predict sorption behaviour of PAHs and other pollutants. Further, Gil-Cardeza et $a .^{42}$ using experimental techniques, have reported that GiHsp60 contributes to the sequestration of chromium(III).

\section{Conclusion}

GiHsp60 has been termed as a miracle protein for soil sustainability. However, it is an unusual molecule that has proven difficult to analyse biochemically due to its recalcitrance and complexity ${ }^{40}$. GiHsp60 not only facilitates root penetration, but also binds to the soil, preventing loss of water from the soil exposed to various abiotic stresses ${ }^{43}$; thereby, regulating the water relations within the soil-plant continuum. The present study thus opens up avenues for undertaking further studies in understanding the role of GiHsp60 in the amelioration of contaminated soil, thereby improving soil fertility. With the role of GiHsp60 clearly established in soil water aggregation ${ }^{7}$, the predicted model and its interactions with SOM can further encourage biotechnological research. The study can offer immense potential in exploring the applications of GiHsp60 in water-starved soil, especially in countries which have been witnessing growing water scarcity over the years.

Conflicts of interest: The authors declare no conflict of interest.

1. Kemper, W. D. and Rosenau, R. C., Aggregate stability and size distribution. In Methods of Soil Analysis, Part 1. Physical and Mineralogical Methods, Soil Science Society of America, Madison, Wisconsin, USA, Agronomy Monograph, No. 9, 1986, 2nd edn, pp. 425-442.

2. Lehmann, A., Fitschen, K. and Rillig, M. C., Abiotic and biotic factors influencing the effect of microplastic on soil aggregation. Soil Syst., 2019, 3, 21.

3. Leifheit, E. F., Veresoglou, S. D., Lehmann, A., Morris, E. K. and Rillig, M. C., Multiple factors influence the role of arbuscular mycorrhizal fungi in soil aggregation - a meta-analysis. Plant Soil, 2014, 374, 523-537.

4. Wright, S. F. and Upadhyaya, A., Extraction of an abundant and unusual protein from soil and comparison with hyphal protein of arbuscular mycorrhizal fungi. Soil Sci., 1996, 161, 575586

5. Purin, S. and Rillig, M. C., The arbuscular mycorrhizal fungal protein glomalin: limitations, progress, and a new hypothesis for its function. Pedobiologia, 2007, 51, 123-130.

6. Gadkar, V. and Rillig, M. C., The arbuscular mycorrhizal fungal protein glomalin is a putative homolog of heat shock protein 60 . FEMS Microbiol. Lett., 2006, 263, 93-101.

7. Rillig, M. C., Wright, S. F., Nichols, K. A., Schmidt, W. F. and Torn, M. S., Large contribution of arbuscular mycorrhizal fungi to soil carbon pools in tropical forest soils. Plant Soil, 2001, 233, 167-177.
8. Jia, X., Zhao, Y., He, Y. and Chang, Y., Glomalin-related soil protein in the rhizosphere of Robinia pseudoacacia L. seedlings under higher air temperature combined with Cd-contaminated soil. Eur. J. Soil Sci., 2018, 69, 634-645.

9. Rosier, C. L., Hoye, A. T. and Rillig, M. C., Glomalin-related soil protein: assessment of current detection and quantification tools. Soil Biol. Biochem., 2006, 38, 2205-2211.

10. Mothay, D. and Ramesh, K. V., Evolutionary history and genetic diversity study of heat-shock protein 60 of Rhizophagus irregularis. J. Genet., 2019, 98, 48.

11. Bauer, M. A. et al., CDD: NCBI's conserved domain database. Nucleic Acids Res., 2014, 43(D1), D222-D226.

12. Servant, F., Bru, C., Carrere, S., Courcelle, E., Gouzy, J., Peyruc, D. and Kahn, D., ProDom: automated clustering of homologous domains. Brief Bioinform., 2002, 3, 246-251.

13. Thompson, J. D., Higgins, D. G. and Gibson, T. J., CLUSTAL W: improving the sensitivity of progressive multiple sequence alignment through sequence weighting, position-specific gap penalties and weight matrix choice. Nucleic Acids Res., 1994, 22, 4673 4680 .

14. Bailey, T. L. et al., MEME SUITE: tools for motif discovery and searching. Nucleic Acids Res., 2009, 20, W202-W208.

15. Jonassen, I., Efficient discovery of conserved patterns using a pattern graph. Bioinformatics, 1997, 13, 509-522.

16. Mizuguchi, K., Deane, C. M., Blundell, T. L., Johnson, M. S. and Overington, J. P., JOY: protein sequence-structure representation and analysis. Bioinformatics, 1998, 14, 617-623.

17. Gupta, R., Jung, E. and Brunak, S., Prediction of $N$-glycosylation sites in human proteins. In preparation, 2004, pp. 203-206.

18. Schwede, T., Kopp, J., Guex, N. and Peitsch, M. C., SWISSMODEL: an automated protein homology-modeling server. Nucleic Acids Res., 2003, 31, 3381-3385.

19. Johansson, M. U., Zoete, V., Michielin, O. and Guex, N., Defining and searching for structural motifs using DeepView/SwissPdbViewer. BMC Bioinform., 2012, 13, 173.

20. Fiser, A. and Do, R. K. G., Modeling of loops in protein structures. Protein Sci., 2000, 9, 1753-1773.

21. Fiser, A. and Sali, A., ModLoop: automated modeling of loops in protein structures. Bioinformatics, 2003, 19, 2500-2501.

22. Eisenberg, D., Lüthy, R. and Bowie, J. U., VERIFY3D: assessment of protein models with three-dimensional profiles. In Methods in Enzymology, Academic Press, Massachusetts, United States, 1997, vol. 277, pp. 396-404.

23. Wiederstein, M. and Sippl, M. J., ProSA-web: interactive web service for the recognition of errors in three-dimensional structures of proteins. Nucleic Acids Res. (Suppl. 2), 2007, 35, W407W410.

24. Laskowski, R. A., MacArthur, M. W., Moss, D. S. and Thornton, J. M., PROCHECK: a program to check the stereochemical quality of protein structures. J. Appl. Crystallogr., 1993, 26, 283-291.

25. Schulten, H. R. and Schnitzer, Chemical model structures for soil organic matter and soils. Soil Sci., 1997, 162, 115-130.

26. Barak, P. and Nater, E., The Virtual Museum of Minerals and Molecules, San Fransisco, 1999; http://www.soils.wisc.edu/ virtual_museum/

27. Frisch, M. J. et al., Gaussian 09, Revision D. 01, Gaussian, Inc, Wallingford, CT, USA, 2009.

28. Dennington, R. D. I. I., Keith, T. and Millam, J., GaussView, Version 4.1.2, Semichem Inc, Shawnee Mission, KS, USA, 2007.

29. Case, D. A. et al., Amber 10 (No. BOOK), University of California, USA, 2008.

30. Pettersen, E. F., Goddard, T. D., Huang, C. C., Couch, G. S., Greenblatt, D. M., Meng, E. C. and Ferrin, T. E., UCSF Chimera a visualization system for exploratory research and analysis. J. Comput. Chem., 2004, 25, 1605-1612.

31. Allen, W. J. et al., DOCK 6: impact of new features and current docking performance. J. Comput. Chem., 2015, 36, 1132-1156. 
32. DeLano, W. L., Pymol: an open-source molecular graphics tool. CCP4 Newsl. Protein Crystallogr., 2002, 40, 82-92.

33. Dolinsky, T. J., Nielsen, J. E., McCammon, J. A. and Baker, N. A., PDB2PQR: an automated pipeline for the setup of PoissonBoltzmann electrostatics calculations. Nucleic Acids Res. (Suppl. 2), 2004, 32, W665-W667.

34. Ooi, T., Oobatake, M., Nemethy, G. and Scheraga, H. A., Accessible surface areas as a measure of the thermodynamic parameters of hydration of peptides. Proc. Natl. Acad. Sci. USA, 1987, 84, 3086-3090.

35. Lin, Z., Schwarz, F. P. and Eisenstein, E., The hydrophobic nature of GroEL-substrate binding. J. Biol. Chem., 1995, 270, 10111014.

36. Fernandes, F. F., Rossetti, R. A. M., Coelho-Castelo, A. A. M. and Panunto-Castelo, A., Molecular modeling of heat shock protein of 60-Kda from Paracoccidioides brasiliensis: the first in silico structural model of a fungal Hsp60. J. Comput. Sci. Syst. Biol., 2014, 8, 241-244.

37. Rabe, M., Verdes, D. and Seeger, S., Understanding protein adsorption phenomena at solid surfaces. Adv. Colloid Interface Sci. 2011, 162, 87-106

38. Perry IV, T. D., Cygan, R. T. and Mitchell, R., Molecular models of alginic acid: Interactions with calcium ions and calcite surfaces. Geochim. Cosmochim. Acta, 2006, 70, 3508-3532.

39. Wu, Z., McGrouther, K., Huang, J., Wu, P., Wu, W. and Wang, H., Decomposition and the contribution of glomalin-related soil protein (GRSP) in heavy metal sequestration: field experiment. Soil Biol. Biochem., 2014, 68, 283-290.
40. Wright, S. F. and Anderson, R. L., Aggregate stability and glomalin in alternative crop rotations for the central Great Plains. Biol. Fertil. Soils, 2000, 31, 249-253.

41. Saparpakorn, P., Kim, J. and Hannongbua, S., Investigation on the binding of polycyclic aromatic hydrocarbons with soil organic matter: a theoretical approach. Molecules, 2007, 12, 703-715.

42. Gil-Cardeza, M. L., Ferri, A., Cornejo, P. and Gomez, E., Distribution of chromium species in a Cr-polluted soil: presence of $\mathrm{Cr}(\mathrm{III})$ in glomalin related protein fraction. Sci. Total Environ., 2014, 493, 828-833.

43. Zou, Y. N., Srivastava, A. K., Wu, Q. S. and Huang, Y. M., Glomalin-related soil protein and water relations in mycorrhizal citrus (Citrus tangerina) during soil water deficit. Arch. Agron. Soil Sci., 2014, 60, 1103-1114.

ACKNOWLEDGEMENT. We thank Jain (deemed to be University), Bengaluru for providing the necessary facilities to carry out this work and for providing research scholarship to Ms Dipti Mothay.

Received 9 January 2020; revised accepted 17 October 2020

doi: $10.18520 / \mathrm{cs} / \mathrm{v} 120 / \mathrm{i} 2 / 389-397$ 